\title{
QUALITY-ADJUSTED LIFE EXPECTANCY
}

\author{
Ruut Veenhoven
}

In: Alex C. Michalos (Ed.) Encyclopedia of Quality of Life and Well-Being Research Springer, Dordrecht, Netherlands, 2014. Springer Reference Series, pp. 5320-5321. ISBN 978-94-007-0752-8

DOI: $10.1007 / 978-94-007-0753-5 \_2386$

\section{SYNONYMS}

Disability-adjusted life years (DALY); Happy life expectancy; Happy life years (HLY); Health-adjusted life years (HALY); Healthy life expectancy

\section{DEFINITION}

Statistical estimate of how long and well a particular kind of people will live. Combination of (a) length of life and (b) quality of life.

\section{DESCRIPTION}

\subsection{Concept}

The term life expectancy is used for statistical estimates of how long a particular kind of people will live. Such estimates are based on the observed length of life of similar people who have died in the past and on probable future changes in mortality. Used in this sense, the term refers to something "objective," that is, it is an estimate made by distant observers on the basis of clear criteria. The term is typically not used for subjective appraisals, that is, guesses made by individuals of how long they will live.

These statistical estimates can concern the general population in a particular country, for instance, the life expectancy at birth in Denmark in 2008 was 77.9. Such estimates are also made for people working in particular occupations or for patients suffering from a particular disease.

A long life is not necessarily a good life, for instance, not when medical technology is used to postpone death without stopping a patients suffering. Hence, there is a need for estimates that do not limit to the length of life, but also take its quality into account. There are different notions of what marks quality of life, and different measures of quality-adjusted life expectancy have been developed along these notions. Most of these involve subjective appraisals of one's personal life, which 
means that estimates of quality-adjusted life expectancy involve typically a mix of both objective and subjective assessments.

\subsection{Measures}

The first measures of quality-adjusted life expectancy were developed for use in the field of health care. In this context, "quality of life" is equated with "health" in a broad sense and is typically measured using questionnaires on which patients report how they are doing health-wise. Such questionnaires address various aspects of health, such as limitations to functioning and emotional reactions. The responses are then summed in an index which is used as an indicator of what is called "health-related quality of life" (Sassi, 2006). When combined with observed lifetime, the measures are used to compute "quality-adjusted life years", abbreviated as QALYs. A better name is healthadjusted quality of life.

A variant of QOLYs focuses on a particular aspect of poor health, that is, being "disabled." In this context, disability is also often measured using self-reports, now typically focusing on daily activities, such as dressing oneself and climbing stairs. This kind of measures produces estimates of "disability-adjusted life years, abbreviated as DALYs (Amensen \& Nord, 1999). Quality of life is also seen to manifest in subjective enjoyment of life, and in this line measures of "happiness-adjusted life expectancy" have been developed, commonly called "happy life years" and abbreviated as HLYs (Veenhoven, 2011a, b).

\subsection{Uses}

Life expectancy is a central outcome variable in the health sector and figures also as an indicator of societal development. Data on quality- adjusted life expectancy are used in the same way as unadjusted life-expectancy data. In health care, they are used to make medical decisions, both for making more educated guesses about the effects of individual treatments and for evaluating treatments and drugs. Quality-adjusted life expectancy is particularly important in palliative care. Data on quality-adjusted life expectancy are also used to assess the effectiveness of public health systems in regions or nations.

Data on quality-adjusted life expectancy are also used to assess the livability of social institutions. If residents of a care home live long and healthy, the living conditions in that institute are apparently appropriate. Likewise, if the inhabitants of a country live long and happy, the living conditions in that country cannot be too bad. 


\section{REFERENCES}

Amensen, T., \& Nord, E. (1999)

The value of DALY life: Problems with ethics and validity of disability adjusted life years.

BMJ, 319(7222), 1423-1425.

Sassi, F. (2006)

Calculating $Q A L Y$ 's, comparing $Q A L Y$ and DALY calculations.

Health Policy and Planning, 21, 402-408.

Veenhoven, R. (2011a)

Happy life years in nations: How long and happy people live.

World Database of Happiness, Erasmus University, Rotterdam

Veenhoven, R. (2011b).

Trend happy life years in nations.

World Database of Happiness, Erasmus University, Rotterdam

WHO, (2011)

Global burden of disease

\section{Cross-References in Encyclopedia}

Happiness Adjusted Life Years

Livability Theory

Quality of Life 